\title{
Linear dichroism amplification: Adapting a long-known technique for ultrasensitive femtosecond IR spectroscopy
}

\author{
Réhault, Julien ; Zanirato, V ; Olivucci, M ; Helbing, J
}

\begin{abstract}
We demonstrate strong amplification of polarization-sensitive transient IR signals using a pseudo-null crossed polarizer technique first proposed by Keston and Lospalluto [Fed. Proc. 10, 207 (1951)] and applied for nanosecond flash photolysis in the visible by Che et al. [Chem. Phys. Lett. 224, 145 (1994)]. We adapted the technique to ultrafast pulsed laser spectroscopy in the infrared using photoelastic modulators, which allow us to measure amplified linear dichroism at kilohertz repetition rates. The method was applied to a photoswitch of the N-alkylated Schiff base family in order to demonstrate its potential of strongly enhancing sensitivity and signal to noise in ultrafast transient IR experiments, to simplify spectra and to determine intramolecular transition dipole orientations.
\end{abstract}

DOI: https://doi.org/10.1063/1.3572334

Posted at the Zurich Open Repository and Archive, University of Zurich ZORA URL: https://doi.org/10.5167/uzh-49633

Journal Article

Published Version

Originally published at:

Réhault, Julien; Zanirato, V; Olivucci, M; Helbing, J (2011). Linear dichroism amplification: Adapting a long-known technique for ultrasensitive femtosecond IR spectroscopy. Journal of Chemical Physics, 134(12):124516.

DOI: https://doi.org/10.1063/1.3572334 


\title{
Linear dichroism amplification: Adapting a long-known technique for ultrasensitive femtosecond IR spectroscopy
}

\author{
Julien Réhault, ${ }^{1}$ Vinicio Zanirato, ${ }^{2}$ Massimo Olivucci, ${ }^{3}$ and Jan Helbing ${ }^{1, a)}$ \\ ${ }^{1}$ Physikalisch-Chemisches Institut, Universität Zürich, Winterthurerstrasse 190, 8057 Zürich, Switzerland \\ ${ }^{2}$ Dipartimento di Scienze Farmaceutiche, Universitá di Ferrara, via Fossato di Mortara 17-19, \\ I-44100 Ferrara, Italy \\ ${ }^{3}$ Dipartimento di Chimica, Universitá di Siena, via Aldo Moro 2, I-53100 Siena, Italy \\ and Chemistry Department, Bowling Green State University, Bowling Green, Ohio 43403, USA
}

(Received 31 January 2011; accepted 10 March 2011; published online 30 March 2011)

\begin{abstract}
We demonstrate strong amplification of polarization-sensitive transient IR signals using a pseudonull crossed polarizer technique first proposed by Keston and Lospalluto [Fed. Proc. 10, 207 (1951)] and applied for nanosecond flash photolysis in the visible by Che et al. [Chem. Phys. Lett. 224, 145 (1994)]. We adapted the technique to ultrafast pulsed laser spectroscopy in the infrared using photoelastic modulators, which allow us to measure amplified linear dichroism at kilohertz repetition rates. The method was applied to a photoswitch of the N-alkylated Schiff base family in order to demonstrate its potential of strongly enhancing sensitivity and signal to noise in ultrafast transient IR experiments, to simplify spectra and to determine intramolecular transition dipole orientations. (C) 2011 American Institute of Physics. [doi:10.1063/1.3572334]
\end{abstract}

\section{INTRODUCTION}

In ultrafast transient absorption spectroscopy, valuable information is obtained by comparing the signals measured with parallel and perpendicular polarizations of the pump and the probe pulse. ${ }^{1}$ The difference between these signals can be related directly to the angle between the pumped and the probed transition dipole moments. In the mid-IR at early pump-probe delays (before any reorientation takes place), anisotropy experiments thus provide a powerful tool for probing molecular structure, ${ }^{2,3}$ in particular in combination with quantum chemistry calculations. On a longer time scale, the decay of the polarization-dependence of the transient signals can be related to diffusion constants that depend on the shape of the molecule and its interaction with the environment. . $^{45}$ Linear dichroism (LD) and anisotropy are essentially two metrics of the same quantity. LD is usually defined as (Jensen et $a .^{6}$ )

$$
\mathrm{LD}=\frac{\Delta A_{\|}-\Delta A_{\perp}}{2} \ln (10)
$$

while anisotropy is defined by the ratio

$$
a=\frac{\Delta A_{\|}-\Delta A_{\perp}}{\Delta A_{\|}+2 \Delta A_{\perp}}
$$

with $\Delta A_{\|}$and $\Delta A_{\perp}$ being the transient absorption changes measured for parallel and perpendicular polarization of pump and probe pulses, respectively:

$$
\Delta A_{\|}=\log _{10}\left(\frac{I_{\|}^{\text {pump on }}}{I_{\|}^{\text {pump off }}}\right), \quad \Delta A_{\perp}=\log _{10}\left(\frac{I_{\perp}^{\text {pump on }}}{I_{\perp}^{\text {pump off }}}\right) .
$$

\footnotetext{
a)Electronic mail: j.helbing @ pci.uzh.ch.
}

Here we present measurements of ultrafast transient absorption and LD with enhanced sensitivity, adapting a crossed polarizer quasi-null scheme ${ }^{7}$ as employed by Che et al. ${ }^{8}$ in flash photolysis experiments in the visible spectral range. The basic principle of the method is illustrated in Fig. 1.

Transient absorption signals $\Delta A_{\|}$and $\Delta A_{\perp}$ can be measured by changing the probe light polarization (red, Fig. 1) from parallel to perpendicular with respect to the pump-pulse polarization (blue, Fig. 1). In order to calculate each of the two transient signals of Eq. (3), the light intensity $I_{\|}$or $I_{\perp}$ measured by the detector in the presence of the pump pulse is divided by the intensity with the pump pulse blocked. Because of this normalization, the same transient absorption and linear dichroism signals are obtained when parallel and perpendicular polarized pulses are attenuated behind the sample by the same amount, for example, by inserting a horizontally oriented polarizer at $45^{\circ}$ with respect to the pump-polarization direction [see Fig. 1(b)]. Che et al. ${ }^{8}$ demonstrated that the LD signal can be significantly enhanced, when the angle between the two probe polarizations is now reduced from $90^{\circ}$ to a small angle $2 \beta$ as indicated in Fig. 1(c). Explicitly, they showed that

$$
s=\frac{I_{+\beta}-I_{-\beta}}{I_{+\beta}+I_{-\beta}} \approx \frac{\mathrm{LD}}{\tan \beta} .
$$

Linear dichroism measurements in picosecond timeresolved electronic spectroscopy were originally carried out with exactly crossed polarizers $(\beta=0),{ }^{4,5}$ in which case the background-free pump-induced signal $I_{0}$ 。 depends quadratically on LD, but also on linear birefringence (LB). When one of the two polarizers is slightly tilted $(\beta>0)$, a small fraction of the probe light leaks to the detector and can selectively heterodyne the additional field due to pump-induced linear dichroism. ${ }^{9}$ In this language, the signal of Eq. (4) is the 


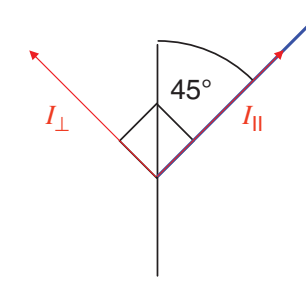

(a)

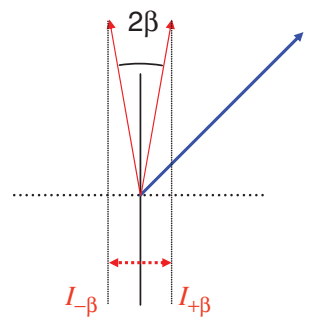

(c)

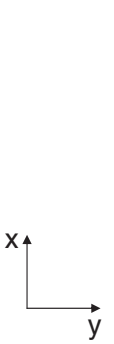

(b)

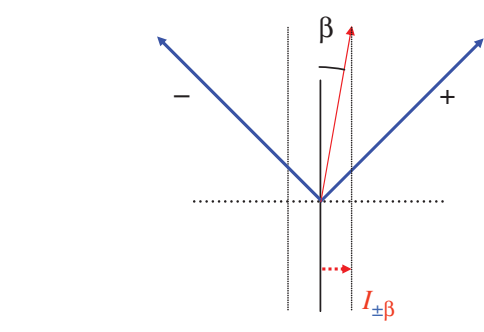

(d)

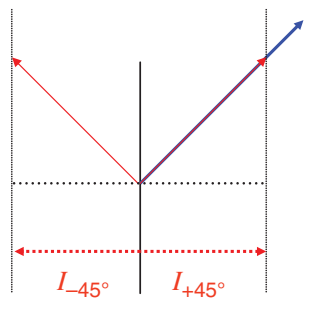

b)
IG. 1. Polarizations of pump (blue) and probe (red) beams in conventional LD and anisotropy measurements (a). The same LD and anisotropy signals can be measured when the horizontal projections of the probe beam is singled out by a polarizer (dotted line) behind the sample (b). They are enhanced when the angle $\beta$ between the vertical axis and the probe polarizations is made smaller (c). Alternatively, the probe polarization can be fixed at an angle $\beta$, and the pump polarization can be varied between $\pm 45^{\circ}$ (d).

difference of two measurements with opposite phases of the heterodyning field. Scherer et al..$^{10}$ could thus study predissociation and wavepacket dynamics of iodine in solution with very high sensitivity.

In this paper we demonstrate how to reliably use this principle in ultrafast pump-probe experiments in the mid-IR. In contrast to earlier implementations of the technique in electronic spectroscopy, which involved separate measurements for each probe polarization, we use fast polarization modulation in order to continuously switch between angles $\pm \beta$ with the help of a photoelastic modulator (PEM), and we explore different experimental arrangements. The advantages of using a PEM to modulate polarization of ultrashort mid-IR laser pulses have been demonstrated earlier in our group in the context of transient vibrational circular dichroism spectroscopy. ${ }^{11}$ Here it allows us to change the linear polarization state of the probe or pump beam at the $\mathrm{kHz}$ repetition rate of our setup and to measure within two successive shots the amplified LD signal. We demonstrate that we can amplify the LD signal by more than 1 order of magnitude without increasing noise. Furthermore, we discuss the possibility to separate overlapping vibrational bands with different anisotropy by recording transient spectra at different polarizer angles. The method also allows us to record highly amplified transient absorption data on time scales shorter than the rotational diffusion time. This makes the crossed polarizer method particularly well-suited for ultrafast vibrational spectroscopy where tiny signals are very common.

\section{EXPERIMENTAL}

An amplified Ti:Sa laser system operating near $800 \mathrm{~nm}$ provided $100 \mathrm{fs}$ pulses of $1 \mathrm{~mJ}$ energy at a repetition rate of $1 \mathrm{kHz}$. Pump pulses at $400 \mathrm{~nm}$ were generated by frequency doubling the fundamental beam, while mid-IR pulses were generated by difference frequency mixing signal and idler beam from a home-built optical parametric amplifier. ${ }^{12}$ The mid-IR pulses with an energy of $2 \mu \mathrm{J}$ covered a frequency range from 1500 to $1700 \mathrm{~cm}^{-1}$ (FWHM) and were focused to a spot size of approximately $100 \mu \mathrm{m}$ at the sample in spatial overlap with the pump beam. After dispersion in a spectrometer their spectral intensity was recorded by a 32 pixel MCT array detector. A small reflection of the mid-IR probe beam from a $\mathrm{BaF}_{2}$ window did not pass polarizers and sample. It was equally dispersed in the monochromator and simultaneously recorded by a second MCT array in order to correct for fluctuations of the probe light. Different spectral intensities of probe and reference beam are normalized out when calculating the signals. Since the grating acts like an additional low extinction ratio polarizer, whenever possible the probe light polarization in the monochromator was kept perpendicular to the lines of the grating, to ensure optimal transmission.

The polarization of either the UV pump or the IR probe laser pulses could be modulated at the repetition rate of the laser system by photoelastic modulators. Since our PEMs need to operate at a given resonance frequency $f \approx 50 \mathrm{kHz}$, we have synchronized the laser to the PEM, as described in detail elsewhere, ${ }^{11}$ and shown schematically in Fig. 2(d). In brief, the fourth harmonic of the PEM frequency was downcounted by an odd number to provide a trigger for the laser amplifier of approximately $1 \mathrm{kHz}$. This trigger was delayed in such a way that the femtosecond pulses cross the modulator when it is in a relaxed state (no polarization change) or at a turning point of its oscillations, i.e., when it acts as a $\pm \lambda / 2$ plate. The linear polarization of probe pulses initially at an angle $\beta$ with respect to the PEM optical axis was thus alternatingly left unchanged or it was rotated to an angle $-\beta$. Three equivalent experimental arrangements of polarizers and PEM are shown in Fig. 2. Part (a) is a direct adaptation of the method used in Ref. 8 and corresponds exactly to the polarization scheme of Fig. 1(c). The UV pump pulse with linear polarization at $45^{\circ}$ with respect to the laboratory $\mathrm{x}$ axis, given by the optical axis of the PEM, excites the sample. It is followed by a mid-IR probe pulse, whose polarization is initially set at an angle $\beta$ by the first polarizer LP1. For every second laser pulse the PEM acts as a $\lambda / 2$ plate and rotates the plane of polarization to $-\beta$. When the sample is isotropic, identical small projections of these probe pulses are transmitted by the second linear polarizer LP2 that is oriented perpendicular to the PEM axis. In practice, it is, however, of advantage to use the arrangement shown in Fig. 2(b), where the order of optical elements in the probe beam is reversed. As shown in the Appendix, this leads to identical transient absorption signals and linear dichroism enhancement. Method (b) facilitates the alignment of LP1 (the polarizer is rotated until the probe pulse polarization coincides exactly with the optical axis of the PEM, i.e., the transmitted intensities for all modulator states in the absence of the pump beam are equal). It also allows us to place the PEM very close to the focal spot of the probe beam (immediately behind the sample), which strongly reduces artifacts arising from lensing effects.

As a third alternative we have also used a PEM for UV and visible light to modulate the pump-pulse polarization between $\pm 45^{\circ}$, keeping the probe polarization fixed as shown 
(a)

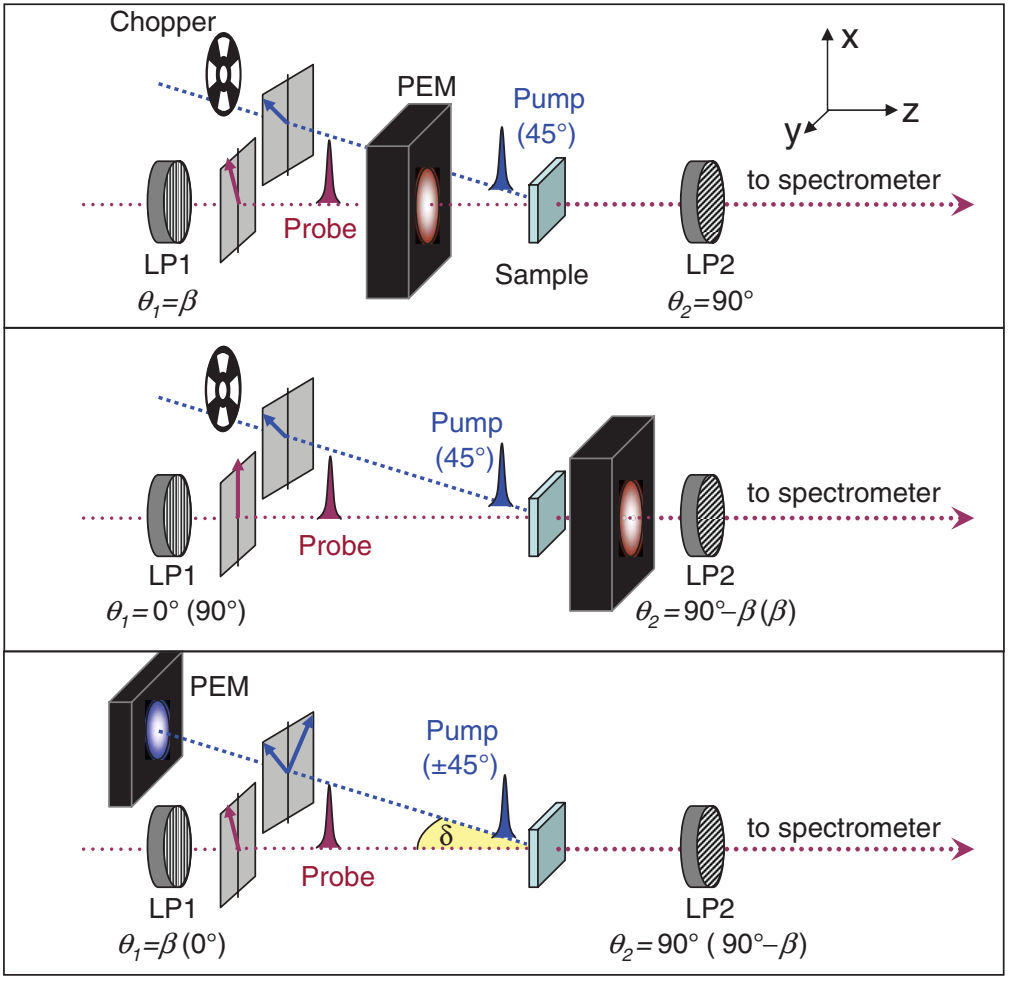

(c)

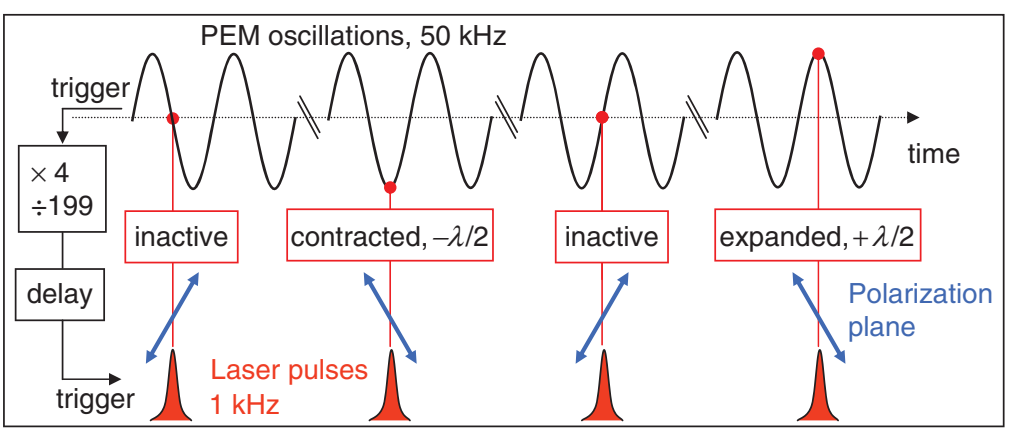

FIG. 2. Three equivalent experimental arrangements for the recording of the enhanced transient linear dichroism signals. (a) Probe beam polarization modulation before the sample, (b) probe beam polarization modulation behind the sample, (c) pump-beam polarization modulation. The orientation $\theta$ of the linear polarizers LP1 and LP2 with respect to the $\mathrm{x}$ axis is indicated below the polarizers, the values in parenthesis correspond to equivalent alternative orientations. Part (d) shows schematically the state of the PEM for four consecutive laser pulses and their planes of polarization.

in Fig. 2(c). This further reduces noise and simplifies the alignment of the probe beam.

\section{SAMPLE}

The sample we used for demonstrating the method is a photoswitch of the N-alkylated Schiff base family ${ }^{13,14}$ [4-(6-methoxy-2,2-dimethyl-3,4-dihydro-2H-naphthalen-1ylidene)-1,5-dimethyl-3,4-dihydro-2H-pyrrolium; see Fig. 3]. The details of its synthesis can be found in the supplementary information. ${ }^{15}$ These photoswitches isomerize from cis (Z) to trans (E) after excitation in the near UV (see Fig. 3, top). Isomerization is ultrafast (return to the ground state in less than $1 \mathrm{ps})$, efficient $(\geq 20 \%)$, and the switches are robust under illumination. ${ }^{16}$ For this $\mathrm{N}$-alkylated Schiff base switch, the $\mathrm{E}$ isomers generated by the pump pulse relax within a few seconds back to $\mathrm{Z}$ at room temperature. The ground state barrier is $22.4( \pm 2) \mathrm{kcal} \mathrm{mol}^{-1}$. We could therefore use this molecule dissolved in deuterated methanol in a flow cell over long time periods without a change in the composition of the sample (nearly $100 \% \mathrm{Z}$ ).

The FTIR spectrum shows two main bands in the spectral range between 1500 and $1700 \mathrm{~cm}^{-1}$. The first one centered at $1566 \mathrm{~cm}^{-1}$ is related to a mode involving mainly stretching of the isomerizing $\mathrm{C}=\mathrm{C}$ bond, and the second one around $1604 \mathrm{~cm}^{-1}$ involves ring modes on the benzene ring (from normal mode calculations on the B3LYP level, GAUSSIAN program suite ${ }^{17}$ ). The shoulders near 1550 and $1620 \mathrm{~cm}^{-1}$ could not be uniquely assigned but are probably related to delocalized modes involving $\mathrm{CH}_{3}$ bending.

\section{RESULTS}

In Fig. 3(b) we present transient absorption spectra $\Delta A_{\|}$ and $\Delta A_{\perp}$ from a conventional (no polarizer in probe beam) measurement with parallel and perpendicular pump and probe pulses at a delay of $500 \mathrm{fs}$. At this delay artifacts due to pulse overlap or a Kerr effect can be excluded. A fraction of 


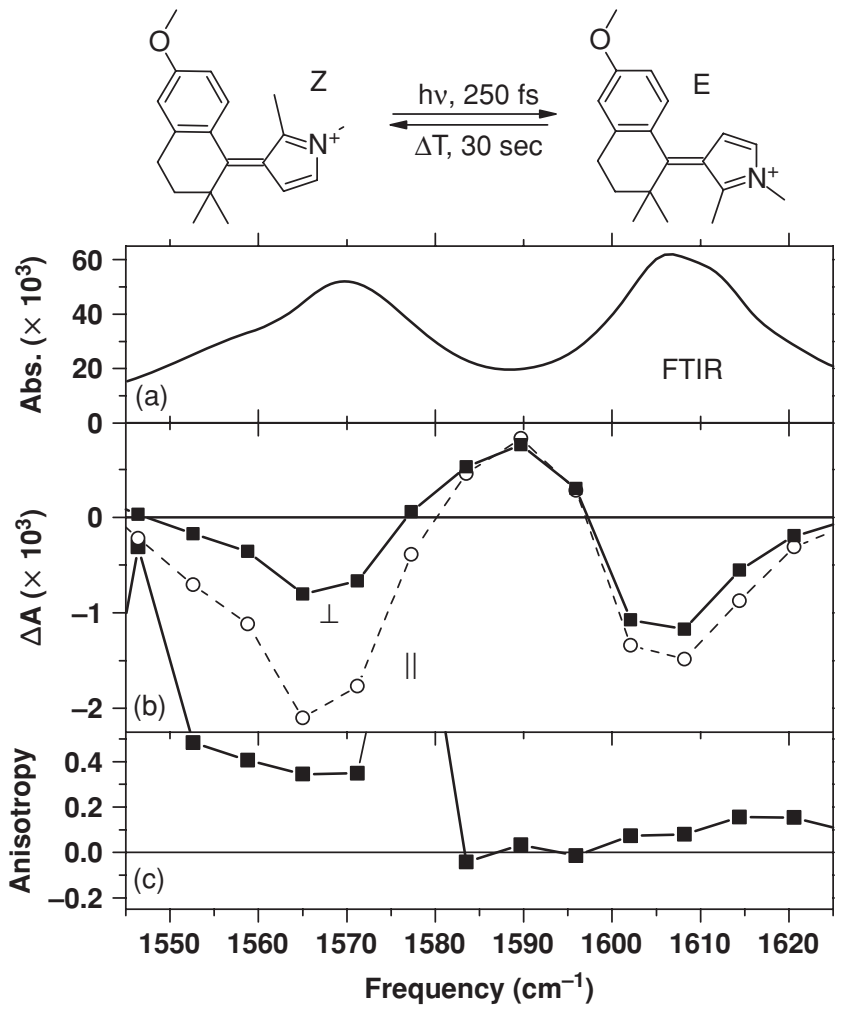

FIG. 3. (a) FTIR spectrum of the Z-isomer of the N-alkylated Schiff base photoswitch. (b) Transient absorption changes $500 \mathrm{fs}$ after excitation at $400 \mathrm{~nm}$ for parallel and perpendicular pump and probe polarizations. (c) Anisotropy calculated from the two spectra in (b).

the molecules has already decayed back to the (hot) electronic ground states, but the bleach of the Z-isomer vibrational bands is almost identical to that seen at earlier delays. Part (c) of the same figure shows the corresponding anisotropies [Eq. (2)]. From these we can calculate the angles between the electronic transition dipole and the IR transition dipole of the individual modes, which are summarized in Table I.

The vibrational transition dipole of the $\mathrm{C}=\mathrm{C}$ stretch mode is nearly parallel to the electronic transition dipole, as expected for a charge translocation across the isomerizing $\mathrm{C}=\mathrm{C}$ bond. ${ }^{13}$ The benzene ring mode has a much smaller anisotropy, yielding an angle around $39^{\circ}-49^{\circ}$. The positive signal around $1585 \mathrm{~cm}^{-1}$ is due to ring modes of molecules in the $S_{1}$ excited state as well as molecules which have already returned to the hot ground state in either the original $\mathrm{Z}$ or the E configuration. The anisotropy of this signal appears to be approximately zero corresponding to an angle near $55^{\circ}$. The anisotropy is increasing between 1590 and $1620 \mathrm{~cm}^{-1}$

TABLE I. Experimental anisotropies, polarizer angles $\left|\beta_{0}\right|$, and corresponding intramolecular angles $\omega$ between the electronic transition dipole moment and the vibrational transition dipole moments.

\begin{tabular}{lccc}
\hline \hline Mode & $\mathrm{C}=\mathrm{C}$ & Photoproduct absorption & Ring mode \\
\hline Frequency & $1566 \mathrm{~cm}^{-1}$ & $1585 \mathrm{~cm}^{-1}$ & $1604 \mathrm{~cm}^{-1}$ \\
Anisotropy & $0.35-0.4$ & $-0.05-0.05$ & $0.06-0.16$ \\
Angles & $0^{\circ}-15^{\circ}$ & $50^{\circ}-60^{\circ}$ & $39^{\circ}-49^{\circ}$ \\
$\left|\beta_{0}\right|$ & $20^{\circ}-25^{\circ}$ & $6^{\circ}-9^{\circ}$ & $11.5^{\circ}-12.5^{\circ}$ \\
Angles & $13^{\circ}-27^{\circ}$ & $44^{\circ}-48^{\circ}$ & $39^{\circ}-41^{\circ}$ \\
\hline \hline
\end{tabular}
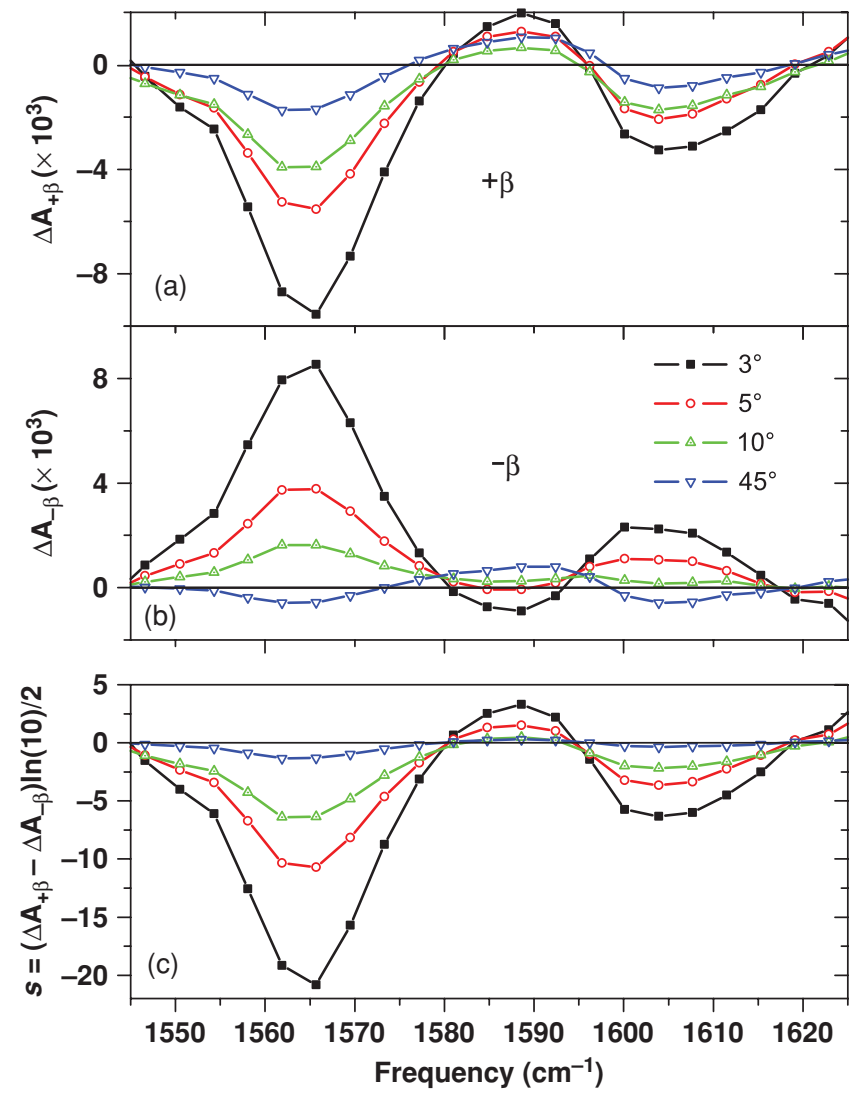

FIG. 4. (a) and (b) Amplified transient absorption signals $\Delta A_{ \pm \beta}$ for different probe angles (polarizer orientations) $\beta$, recorded using setup Fig. 5(b), $500 \mathrm{fs}$ after $400 \mathrm{~nm}$ excitation. (c) Enhanced LD signal $s$.

due to the overlap between this signal and ground state bleaching of the ring mode at $1604 \mathrm{~cm}^{-1}$. Indeed, when two bands with different anisotropies overlap, the anisotropy in the overlapping region can vary between the anisotropy values of the two bands, and can diverge where the pump-probe signal changes sign. Small offsets in these regions can also perturb the measured anisotropy considerably. This is most likely the reason for anisotropy values larger than 0.4 in Fig. 3(c).

In Figs. 4(a) and 4(b) we show the transient signals

$$
\Delta A_{ \pm \beta}=\log _{10}\left(\frac{I_{ \pm \beta}^{\text {pump on }}}{I_{ \pm \beta}^{\text {pump off }}}\right)
$$

at the same pump-probe delay (500 fs), recorded with the setup of Fig. 2(b) for four different analyzer angles between $3^{\circ}$ and $45^{\circ}$. The pump polarization was fixed and pairs of spectra for $\pm \beta$ were measured quasisimultaneously with fast modulation of the probe polarization. The signals for $\beta$ $= \pm 45^{\circ}$ (blue) are very similar to the ones in Fig. 3, obtained with the standard method without polarizers. For smaller analyzer angles, on the other hand, there are significant deviations, due to a different amplification of the different bands as discussed below. In particular, the signal $\Delta A_{+\beta}$ in Fig. 4(a) changes sign, and the higher frequency bands are almost completely eliminated near $\beta=10^{\circ}$ (red). For all angles except $3^{\circ}$, the light intensities on the detector were kept similar by removing filters from the probe beam. For $3^{\circ}$, however, it was approximately two times smaller, leading to a similar signal 

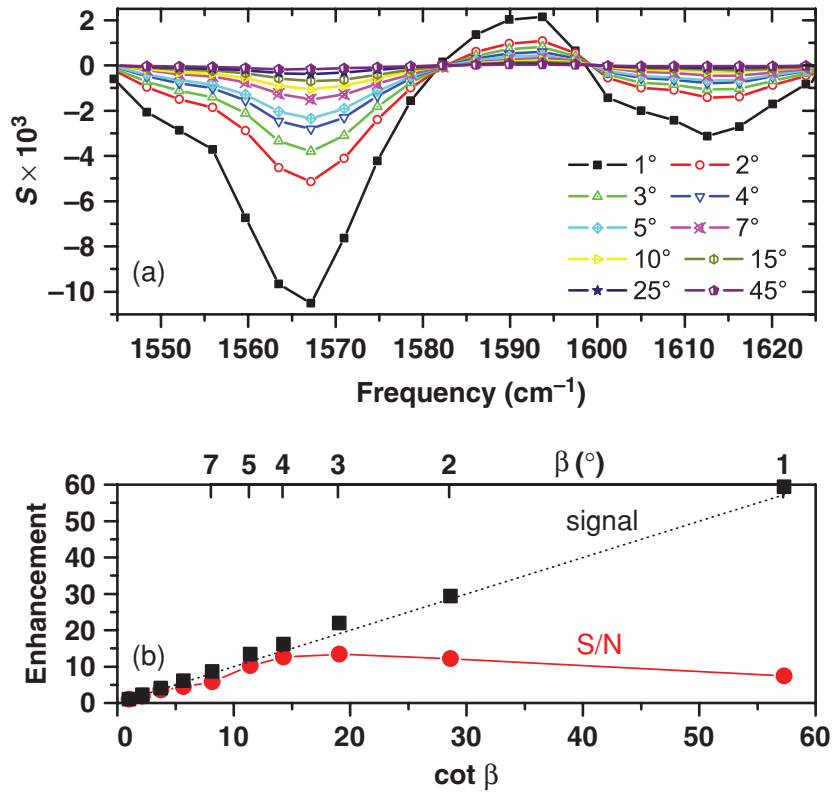

FIG. 5. (a) Enhanced LD signal $s$ recorded by fast modulation of the UV pump pulse polarization [setup (c)], $500 \mathrm{fs}$ after $400 \mathrm{~nm}$ excitation. (b) Enhancement of the signal (squares) and signal to noise ratio $\mathrm{S} / \mathrm{N}$ (circles) as a function of polarizer orientation $\beta$. Both are normalized to 1 at $\beta=45^{\circ}$.

to noise ratio as for $\beta=5^{\circ}$. Figure 4(c) shows the normalized LD signals, given by the difference of the spectra shown in parts (a) and (b). As predicted by Eq. (4) they are identical up to a scaling factor $\cot \beta$.

When only the enhanced LD signal $s$ is needed, it can be measured with even higher sensitivity when the chopper is removed from the pump beam and Eq. (4) is evaluated directly from consecutive measurements with alternating pump or probe polarizations. This is illustrated in Fig. 5, where we show data obtained with the setup of Fig. 2(c), i.e., by modulating the pump-pulse polarization between $\pm 45^{\circ}$ with a fixed horizontal orientation of analyzer LP2 and an incident probe polarization angle $\beta$. For simplicity we still label the corresponding intensities $I_{ \pm \beta}$ as defined in Fig. 1(d). Sample and pump-probe delay are the same as in Figs. 3 and 4, but the UV pump beam was approximately 20 times weaker, in order to minimize saturation effects and reduce the (unamplified) LD close to our noise limit. Without the additional attenuation by the PEM in the mid-IR probe beam, smaller angles $\beta$ between polarizers are possible before the light level at the detector becomes limiting. The plot of the normalized signal to noise ratio at the peak of the main bleach signal near $1566 \mathrm{~cm}^{-1}$ in Fig. 5(b) shows that the data quality could now be improved up to signal amplification factors close to $15\left(\beta=3^{\circ}\right)$.

\section{DISCUSSION}

\section{A. Information content}

Che et $a l .{ }^{8}$ have used the Mueller matrices to evaluate the transmitted intensities $I_{ \pm \beta}$ for a polarization scheme as shown in Fig. 1(c), with a common plane of polarization for pump and probe beams. This leads to the enhanced linear dichroism signal

$$
\begin{aligned}
s & =\frac{I_{+\beta}-I_{-\beta}}{I_{+\beta}+I_{-\beta}} \\
& =\frac{\left(1-r^{2}\right) \sin (2 \beta) \sinh (\mathrm{LD})}{(1+r)^{2} \cosh (\mathrm{LD})-(1-r)^{2} \cos (2 \beta)},
\end{aligned}
$$

where $r$ is the polarizer extinction ratio and LD is the pumpinduced linear dichroism of the sample. From this expression Eq. (4) is obtained by the Taylor expansion, when neglecting the imperfections of the polarizers. As shown in the Appendix, Eq. (6) holds for all three experimental arrangements shown in Fig. 2. However, in mid-IR spectroscopy, where the sample thickness is typically only $100 \mu \mathrm{m}$ or less, pump and probe beams cannot cross at a $90^{\circ}$ angle as in the original flash photolysis experiment. ${ }^{8}$ Neither is it practical to work with collinear UV and IR beams when one needs to precisely control their polarizations independently. As a consequence, there is a small angle $\delta \approx 5^{\circ}$ between the planes of polarization of the pump and the probe beam, which leads to a slightly reduced signal $s_{\delta} \approx \mathrm{LD} \cot \beta \cos \delta$.

LD is related to the intramolecular angle $\omega$ between the pumped and the probed transition dipole moment by the relation (see the Appendix)

$$
\mathrm{LD}=\bar{A}(\omega) \frac{1+3 \cos (2 \omega)}{7+\cos (2 \omega)},
$$

where $\bar{A}$ is the average absorption change for parallel and perpendicular pump and probe pulses (without amplification):

$$
\bar{A}(\omega)=\frac{\Delta A_{\|}+\Delta A_{\perp}}{2} \ln (10)=\frac{\Delta A_{+45}+\Delta A_{-45}}{2} \ln (10),
$$

which is proportional to the number of excited molecules.

Making use of the same approximations ( $r=0$, small LD) we can also compare the transmitted intensities with and without pump excitation and find

$$
\Delta A_{ \pm \beta} \approx \frac{\bar{A}}{\ln (10)}\left(1 \pm \frac{\tan \beta_{0}}{\tan \beta}\right),
$$

with the approximation that $\delta \approx 0$ and where $\left|\beta_{0}\right|$ is the probe polarization angle at which one of the two signals can be eliminated. This angle is given by

$$
\tan \beta_{0}=\frac{\Delta A_{\|}-\Delta A_{\perp}}{\Delta A_{\|}+\Delta A_{\perp}}=\frac{1+3 \cos (2 \omega)}{7+\cos (2 \omega)} .
$$

When $\Delta A_{ \pm \beta}$ is measured at different relative polarizer orientations, the intermolecular angle can thus be determined from the angle at which the signal vanishes, which is independent of the absorbance change. Figure 6 shows linear fits [Eq. (9)] to the peak signals in Fig. 4 as a function of $\cot \beta$; the extracted angles are compared to the analysis of the conventional anisotropy measurements in Table I. Dashed lines in Fig. 6 indicate the signal enhancement for the limiting cases $\omega=0^{\circ}$ and $\omega=90^{\circ}$. When the average absorption change $\bar{A}$ is negative (bleach or stimulated emission), the $\Delta A_{+\beta}$ signal changes sign and grows positive if the angle between transition dipoles is smaller than magic angle $\omega_{\mathrm{mag}} \approx 54.7^{\circ}$; if $\omega>\omega_{\mathrm{mag}}$, on the other hand, $\Delta A_{-\beta}$ changes 


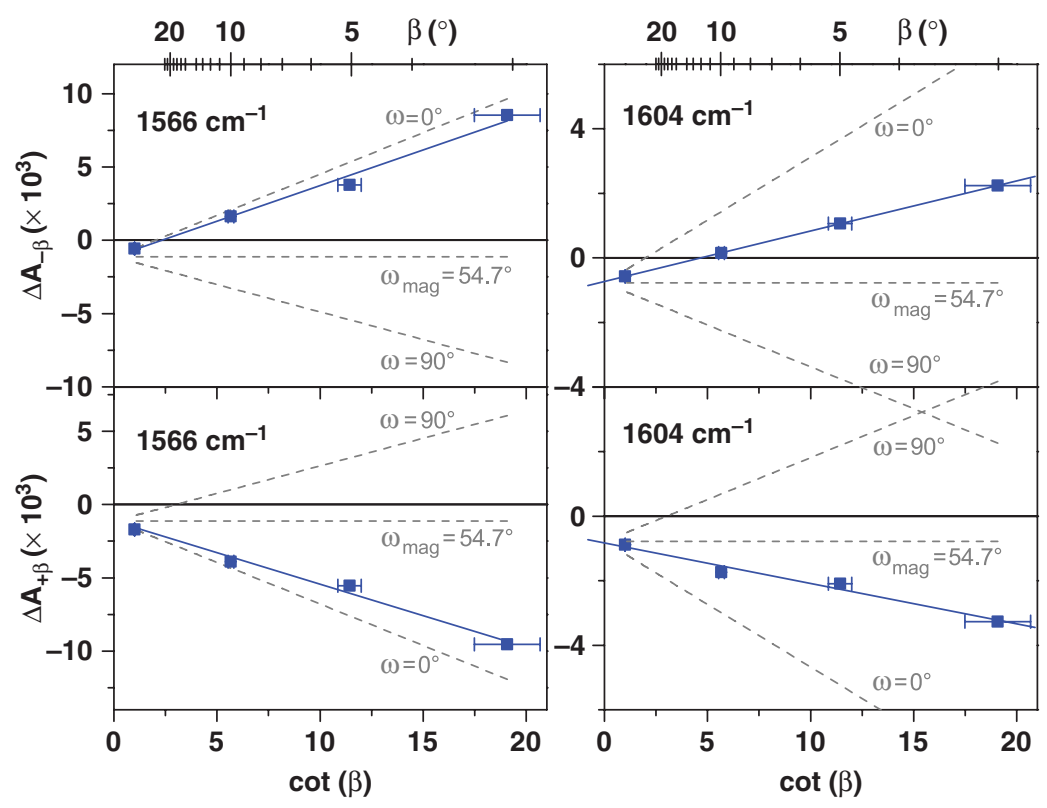

FIG. 6. $\beta$-dependence of the transient absorption signals $\Delta A_{ \pm \beta}$, shown in Fig. 4. Dashed lines show the signals expected for different angles $\omega$ between the pumped and the probed transition dipole moment, assuming the same $\bar{A}$ as for the experimental data.

sign. For a positive absorption change, it is the other way round. Consequently, signals arising from vibrations with a transition dipole moment at magic angle with respect to the electronic transition dipole moment are not enhanced when $\beta$ is reduced (horizontal dashed lines in Fig. 6). This is also the case for isotropic background signals, for example, due to pump-induced temperature changes in the solvent.

The practical advantages of enhanced LD measurements over conventional anisotropy experiments for the determination of intramolecular angles may, however, only be modest. Indeed, in the case of large anisotropies, for example, the $\mathrm{C}=\mathrm{C}$ stretch band, the determination of $\left|\beta_{0}\right|$ or a fit with Eq. (9) depends sensitively on the data quality at relatively large values of $\beta$ or on the average absorbance change $\bar{A}$, i.e., signals recorded with no or rather small amplification. For our test molecule, on the other hand, the $\beta$-dependence of the product absorption signal near $1585 \mathrm{~cm}^{-1}$ is a clear indication of an angle between the corresponding transition dipoles of slightly less than $50^{\circ}$, a result not apparent in the conventional anisotropy data shown in Fig. 3.

Furthermore, for a suitable choice of $\beta=\beta_{0}$, specific absorption bands can be eliminated from the transient spectra $\Delta A_{+\beta}$ or $\Delta A_{-\beta}$, yielding a clearer view of neighboring spectral features or resolving overlapping bands with different anisotropies. This can of course also be achieved by a weighted subtraction of spectra recorded for parallel and perpendicular polarization in conventional anisotropy measurements. With the crossed polarizer techniques, however, band elimination is possible in a single measurement, thereby significantly reducing uncertainties due to noise and background. Also note that fast polarization modulation is not necessary in this case.

To our opinion, however, the most useful signal for ultrafast vibrational spectroscopy is the enhanced linear dichroism signal $s$ itself [Eqs. (4) and (9)], which carries most of the information of regular pump-probe data, but can be measured with more than 1 order of magnitude better signal to noise (or 100 times faster). While this signal decays to zero with rotational diffusion, many of the interesting processes addressed by ultrafast spectroscopy such as photoisomerization, bond-breaking, and intramolecular energy transport take place on a much faster time scale and can be fully captured by the enhanced LD signal. Even much slower processes can be studied in proteins, membranes, or other media that slow down the anisotropy decay. The enhanced LD signal can also be evaluated directly from two consecutive intensity measurements without the need of a chopper in the pump beam. This doubles the effective number of averages in a given time interval, and allows us to fully exploit correlations in laser intensity fluctuations. Indeed, we found that the quality of the LD data recorded without chopper is significantly better than the equivalent difference of the signals $\Delta A_{+\beta}$ and $\Delta A_{-\beta}$, measured quasisimultaneously with a chopper repeatedly blocking and transmitting four consecutive pump pulses, even when the latter data set is averaged twice as long.

\section{B. Limitations}

The implementation of enhanced LD spectroscopy in the mid-IR has so far probably been held back by the poor performance of detectors and the need for sufficiently good polarizers. Detector efficiency is important because the probe light transmitted by the second polarizer is only a small fraction of the incoming light, decreasing from ideally $50 \%$ to $0.3 \%$ when $\beta$ is reduced from $45^{\circ}$ to $3^{\circ}$. In our setup, despite probe pulse energies close to $2 \mu \mathrm{J}$, even lower transmission can no longer be compensated by the removal of filters from the probe beam. Low light intensity at the detector leads to a significant increase in noise and currently limits the signal 
enhancement factor we can achieve to approximately 20-40, depending also on the solvent absorption.

A second limitation in the mid-IR can be the quality of polarizers. Our free-standing wire-grid polarizers (Infraspecs PO3) have an extinction ratio of $10^{-4}$ but suffer from spatial inhomogeneity, which can lead to artifacts. With home-built polarizers based on Brewster angle reflection, ${ }^{18}$ we reach an extinction ratio of $10^{-9}$ but these are bulky and have a maximum transmission of only $38 \%$. More common substrate-supported wire grid polarizers with $r=10^{-2}$, on the other hand, severely limit the maximum possible signal amplification, which occurs at an angle between polarizers $\beta_{\max } \approx \sqrt{2 r}$, corresponding to an enhanced LD signal ${ }^{8}$ $s_{\max } \approx \mathrm{LD} /(2 \sqrt{2 r})$.

When enough light is available and the polarizers are sufficiently good, the possible signal enhancement may depend on the size of the LD itself, as can be seen by evaluating Eq. (6). For example, a (unamplified) LD of $10^{-2}$ could be magnified at most by a factor of approximately 200 $\left(\beta=0.2^{\circ}\right)$ and the signal would change linearly with $\cot \beta$ only up to about $0.5^{\circ}$. This may distort spectra, in particular in electronic spectroscopy, but will rarely be the limiting factor in the mid-IR, where transient absorption changes are typically much smaller.

At very small angles $\beta$, pump-induced linear birefringence may potentially also distort the spectra. Indeed, although LB contributions are not heterodyned and are completely eliminated by taking the difference $I_{+\beta}-I_{-\beta},{ }^{10}$ a quadratic birefringence-dependence of the (amplified) LD signal $s$ is reintroduced in Eq. (6) by the normalization with the sum $I_{+\beta}+I_{-\beta}$. Inclusion of LB in a Mueller matrix analysis leads to a modified equation (6), ${ }^{8}$ where $\cos 2 \beta$ in the denominator is replaced by $\cos 2 \beta \cos \mathrm{LB}$. To lowest order in LB and LD Eq. (4) then becomes

$$
s \approx \mathrm{LD} \cot \beta\left(1-\frac{\cot ^{2} \beta-1}{4} \mathrm{LB}^{2}\right),
$$

which is equivalent to $\ln (10) / 2$ times the difference between the signals

$$
\Delta A_{ \pm \beta} \approx \frac{1}{\ln (10)}\left[\bar{A} \pm \mathrm{LD} \cot \beta\left(1-\frac{\cot ^{2} \beta-1}{4} \mathrm{LB}^{2}\right)\right] .
$$

This means that LB has a negligible effect $(<1 \%)$ on our signals as long as $\beta \geq 5 \mathrm{LB}$. Even in case of an unusually large pump-induced LB of $10^{-2}$ undistorted measurements would be possible down to $\beta=3^{\circ}$.

Finally, from the amplified LD signal alone one cannot distinguish between population relaxation and reorientation dynamics. However, it is possible to completely eliminate the angle-dependence of the signal $\Delta A_{\beta_{M}}$ by setting $\tan \beta_{M}=1 / 3$ (see the Appendix), the so-called mystic angle condition. ${ }^{9}$ Population dynamics can thus be measured independently with the same setup, however, without benefiting from signal amplification.

\section{Fast polarization modulation}

The measurements presented here do not necessarily require the fast switching of pump or probe polarization states by a PEM and can be realized with much less experimental effort without it. Nevertheless, fast polarization modulation affords a significant signal to noise improvement already in conventional anisotropy measurements. At the same time, however, a PEM can lead to additional artifact signals, in particular when placed into the probe beam as in setups (a) and (b) of Fig. 2.

First, a retardation of $\lambda / 2$ can be produced by the PEM only for one specific wavelength, at higher or lower frequencies the polarization state will be slightly elliptical. This is less critical for the visible or UV pump pulses which typically have a much narrower relative bandwidth. Second, a small static birefringence of the PEM breaks the symmetry between $\pm \lambda / 2$ modulation, corresponding to maximum expansion or contraction of the modulator crystal. Since in both states the PEM ideally rotates an incoming polarization into the same plane, we compensate for this effect by averaging the corresponding signals. This reduces the chopper frequency for measurements of $\Delta A_{ \pm \beta}$ to one-eighth of the laser repetition rate, as four signals [PEM inactive, expanded, contracted, and inactive; see Fig. 2(d)] have to be recorded consecutively. Since the refractive index changes in a PEM crystal peak at the center and drop to zero at the edges, at maximum expansion and contraction the PEM also acts as a positive and negative lens, respectively, with a focal length of $60-80 \mathrm{~m}$ (depending on the size of the crystal). The size of the beam at the array detector is thereby modulated, which can lead to intensity changes and spectral shifts. Placing the PEM close to the focal point of the mid-IR probe beam strongly reduces this effect [one of the main advantages of the arrangement in Fig. 2(b) over that in Fig. 2(a)]. In contrast, the corresponding modulation of the focal spot size of the UV pump beam at the sample in the experimental arrangement shown in Fig. 2(c) has a negligible effect on the data, especially after averaging the signals recorded with $\pm \lambda / 2$ modulation.

In summary, fast modulation of the pump-pulse polarization is clearly superior to modulating the mid-IR probe light in crossed polarizer enhanced ultrafast LD spectroscopy. On the other hand, the setup (b) in Fig. 2 can be directly used for (transient) vibrational circular birefringence measurements ${ }^{19,20}$ (for which it was originally designed) by aligning the planes of polarization of pump and probe beams. Our technique for modulating the polarization of mid-IR pulses will also be useful in analogous all-IR pump-probe or 2D-IR experiments. ${ }^{21}$ A closely related experiment has in fact already been performed by Xiong and Zanni, who could enhance signals significantly by placing a polarizer into the probe beam in a 2D-IR experiment with collinear pump beams of perpendicular polarization. ${ }^{22}$

\section{CONCLUSION}

We have demonstrated different experimental arrangements for measuring transient absorption changes and LD in ultrafast mid-IR pump-probe experiments with highly 
improved sensitivity, using a quasi-null crossed polarizer method. Signal amplification by more than 1 order of magnitude was achieved at constant noise. Even larger amplification factors, as have been previously reported for electronic spectroscopy, are possible but require both better detectors or higher probe pulse intensities and polarizers with extinction ratios better than $10^{-4}$.

The crossed polarizer technique is particularly powerful when combined with fast polarization modulation using photoelastic modulators. Equivalent signals can be measured with either pump or probe polarization modulation; however, the latter is more sensitive to artifacts. Here we used both to detect enhanced LD signals after the photoexcitation of a photoswitch of the N-alkylated Schiff base family and determined the angles between electronic and vibrational transition dipole moments with improved accuracy. It was equally possible to eliminate individual spectral features at certain relative polarizer angles in a single measurement.

We expect the technique to become most useful for the amplification of transient signals from samples exhibiting very low absorbance changes. Since signal amplification is proportional to the pump-induced linear dichroism, only bands with a well-defined orientation of their transition dipole moment are significantly enhanced, while solvent and other background signals as well as birefringence contribution are suppressed. This will strongly facilitate the study of light-induced structural changes at much lower concentrations than typically required in conventional transient mid-IR spectroscopy, a perspective particularly attractive for biological samples. Extension of the same measurement principle to other frequency domains as well as multidimensional spectroscopies are possible.

\section{ACKNOWLEDGMENTS}

This work is supported by the Swiss National Science Foundation [NSF(CH)] Grant No. 200020-119814/1.

\section{APPENDIX: EQUIVALENCE OF THE DIFFERENT SETUPS ANGLE-DEPENDENCE AND EFFECTS OF NONCOLLINEAR GEOMETRY}

In order to derive the equations appearing in the text, we first make the approximation of a common plane of polarization for pump and probe pulses. Equation (6) can then be obtained by the Mueller matrix calculus, ${ }^{6}$ as already presented in Ref. 8. The full Mueller matrix for all optical elements in the probe beam as arranged in Fig. 2(a) is given by

$$
M_{a}^{ \pm}=M_{\mathrm{LP}}\left(\theta_{2}\right) M_{\text {sample }} M_{\mathrm{PEM}}^{ \pm} M_{\mathrm{LP}}\left(\theta_{1}\right)
$$

with the Mueller matrices

$$
M_{\mathrm{LP}}(\theta)=\left(\begin{array}{cccc}
\frac{(1+r)}{2} & \frac{q \cos (2 \theta)}{2} & \frac{q \sin (2 \theta)}{2} & 0 \\
\frac{q \cos (2 \theta)}{2} & \frac{2 \sqrt{r}+b \cos (2 \theta)^{2}}{2} & \frac{b \cos (2 \theta) \sin (2 \theta)}{2} & 0 \\
\frac{q \sin (2 \theta)}{2} & \frac{b \cos (2 \theta) \sin (2 \theta)}{2} & \frac{2 \sqrt{r}+b \sin (2 \theta)^{2}}{2} & 0 \\
0 & 0 & 0 & \sqrt{r}
\end{array}\right)
$$

describing the linear polarizers with extinction ratio $r$ $\left.(q=1-r), b=(1-\sqrt{r})^{2}\right)$ at angles with $\theta_{1}=\beta$ and $\theta_{2}=$ $90^{\circ}$ with respect to the $\mathrm{x}$ axis, and

$$
\begin{aligned}
M_{\text {sample }} & =M_{\mathrm{LD}}\left(45^{\circ}\right) \\
& =e^{-\bar{A}}\left(\begin{array}{cccc}
\cosh (\mathrm{LD}) & 0 & \sinh (\mathrm{LD}) & 0 \\
0 & 1 & 0 & 0 \\
\sinh (\mathrm{LD}) & 0 & \cosh (\mathrm{LD}) & 0 \\
0 & 0 & 0 & 1
\end{array}\right)
\end{aligned}
$$

describing a medium with (pump-induced) linear dichroism $\mathrm{LD}$ when the pump polarization is set to $45^{\circ}$. Without the pump beam $\mathrm{LD}=0$ and $\bar{A}=0$. When the PEM is in its equilibrium state (measurement of $I_{+\beta}$ ) it is described by the unity matrix. In the expanded or contracted state (measurement of $I_{-\beta}$ ) it acts as a birefringent $\lambda / 2$ plate oriented in the $\mathrm{x}$ direction, i.e.,

$$
M_{\mathrm{PEM}}^{ \pm}=\left(\begin{array}{cccc}
1 & 0 & 0 & 0 \\
0 & 1 & 0 & 0 \\
0 & 0 & \pm 1 & 0 \\
0 & 0 & 0 & \pm 1
\end{array}\right) .
$$

The transmitted intensity is given by the scalar product of the first row of $M_{a}$ with the Stokes vector of the incoming light: ${ }^{6}$

$$
I_{ \pm \beta}=\left(M_{a}^{ \pm} \cdot \vec{V}_{\text {in }}\right)_{1} .
$$

When the enhanced LD signal is evaluated for an initially unpolarized beam $\vec{V}_{\text {in }}=(1,0,0,0)$, only the first diagonal element is important and we obtain Eq. (6). For a highly polarized incident laser beam, corrections to this expression can be readily calculated. However, for sufficiently good polarizers this only leads to a rescaling of all amplitudes and is normalized out.

The setup (b) in Fig. 2 (with the polarizer orientations $\theta_{1}=90^{\circ}$ and $\theta_{2}=\beta$ given in parentheses) is obtained from setup (a) by reversal of the probe beam propagation direction, and the Mueller matrix $M_{b}$ is given by the product of the same individual matrices in reverse order. Since they are all symmetric, it follows that $M_{b}$ is the transpose of $M_{a}$, and we thus obtain the same transmitted intensities for an unpolarized incident light field or in the limit of good polarizers. Polarizer orientations $\theta_{1}=0^{\circ}$ and $\theta_{2}=90^{\circ}-\beta$ correspond to an exchange of $x$ and $y$ axes.

The full Mueller matrix for setup (c) in Fig. 2 is given by

$$
M_{c}^{ \pm}=M_{\mathrm{LP}}\left(90^{\circ}\right) M_{\text {sample }}^{ \pm} M_{\mathrm{LP}}(\beta),
$$

where $M_{\text {sample }}^{ \pm}=M_{\mathrm{LD}}\left( \pm 45^{\circ}\right)=M_{ \pm \mathrm{LD}}\left(45^{\circ}\right)$ for the pump beam at $\pm 45^{\circ}$ now corresponds to the two measurements $I_{ \pm \beta}$. The equivalence with setup (a) is trivial for the measurement of $I_{+\beta}$, for which the PEM is in an inactive state. For $I_{-\beta}$ measurements the matrix calculus again only shows slight differences between this setup and the others for imperfect polarizers when the incident beam is polarized. The polarizer orientations given in parentheses can again be understood as a reversal of the probe beam direction.

In the limit of perfect polarizers we obtain from Eq. (A5) to linear order in $\bar{A}$ and LD

$$
\frac{I_{ \pm \beta}^{\text {pump on }}}{I_{ \pm \beta}^{\text {pump off }}}=1-\bar{A} \mp \mathrm{LD} \cot \beta
$$


or after taking the decadic logarithm:

$$
\begin{aligned}
\Delta A_{ \pm \beta} & =\frac{1}{\ln (10)}(\bar{A} \pm \mathrm{LD} \cot \beta) \\
& =\frac{1}{2}\left(\left(\Delta A_{\|}+\Delta A_{\perp}\right) \pm\left(\Delta A_{\|}-\Delta A_{\perp}\right) \cot \beta\right) .
\end{aligned}
$$

Pump-induced linear birefringence $\mathrm{LB}=2 \pi\left(\Delta n_{\|}\right.$ $\left.-\Delta n_{\perp}\right) L / \lambda(\lambda=$ wavelength, $L=$ pathlength $)$ can be included in the analysis by setting 6

$$
\begin{aligned}
& M_{\text {sample }} \\
& \quad=e^{-\bar{A}}\left(\begin{array}{lccc}
\cosh (\mathrm{LD}) & 0 & \sinh (\mathrm{LD}) & 0 \\
0 & \cos (\mathrm{LB}) & 0 & \sin (\mathrm{LB}) \\
\sinh (\mathrm{LD}) & 0 & \cosh (\mathrm{LD}) & 0 \\
0 & -\sin (\mathrm{LB}) & 0 & \cos (\mathrm{LB})
\end{array}\right) .
\end{aligned}
$$

For small signals and ideal polarizers Eq. (A8) can also be derived using response theory, which shows that the pumpinduced absorption changes are proportional to the isotropic orientational average ${ }^{23}$

$$
\left\langle\Gamma_{\text {detect }} \Gamma_{\text {probe }} \Gamma_{\text {pump }} \Gamma_{\text {pump }}\right\rangle /\left\langle\Gamma_{\text {detect }} \Gamma_{\text {probe }}\right\rangle .
$$

The vectors $\Gamma$ represent the polarization directions of pump and probe beams at the sample and $\Gamma_{\text {detect }}$ is the polarization component of the probe beam that is detected. When pump and probe beam share the same $X Y$ plane of polarization, the second polarizer in the probe beam is horizontal $\left(\Gamma_{\text {detect }}\right.$ $=Y$ ) and the pump-pulse polarization is at $\pm 45^{\circ}$, i.e., $\Gamma_{\text {pump }}$ $=(X+Y) / \sqrt{2}$ as indicated in Fig. 1(d), and we obtain

$$
\begin{aligned}
\Delta A_{ \pm \beta} \propto & \frac{\left\langle Y(X \cos \beta+Y \sin \beta) \frac{1}{2}(X \pm Y)^{2}\right\rangle}{\langle Y(X \cos \beta+Y \sin \beta)\rangle} \\
\propto & (\langle Y Y Y Y\rangle+\langle Y Y X X\rangle) \\
& \pm(\langle Y X Y X\rangle+\langle Y X X Y\rangle) \cot \beta \\
= & (\langle Y Y Y Y\rangle+\langle Y Y X X\rangle) \\
& \pm(\underbrace{(\langle Y Y Y\rangle}_{\propto \Delta A_{\|}}-\underbrace{\langle Y Y X X\rangle}_{\propto \Delta A_{\perp}}) \cot \beta,
\end{aligned}
$$

where we have used the fact that the orientational averages vanish unless they contain only even numbers of the same polarization direction, as well as the identity $\langle Y X Y X\rangle$ $+\langle Y X X Y\rangle=\langle Y Y Y Y\rangle-\langle Y Y X X\rangle$.

Applying the same rules, it is straightforward to evaluate the signals when pump and probe beams intersect at the sample at an angle $\delta$ and therefore have different planes of polarization. With the substitutions

$$
\begin{aligned}
X_{\text {pump }} & =X_{\text {probe }} \\
Y_{\text {pump }} & =Y_{\text {probe }} \cos (\delta)-Z_{\text {probe }} \sin (\delta) \\
Z_{\text {pump }} & =Y_{\text {probe }} \sin (\delta)+Z_{\text {probe }} \cos (\delta),
\end{aligned}
$$

the pump-induced absorption changes become

$$
\begin{aligned}
\Delta A_{ \pm \beta} \propto & \frac{\left\langle Y(X \cos \beta+Y \sin \beta) \frac{1}{2}(X+[Y \cos \delta-Z \sin \delta])^{2}\right\rangle}{\langle Y(X \cos \beta+Y \sin \beta)\rangle} \\
\propto & \left(\langle Y Y Y Y\rangle \cos ^{2} \delta+\langle Y Y X X\rangle+\langle Y Y Z Z\rangle \sin ^{2} \delta\right) \\
& \pm \cot \beta(\langle Y X Y X\rangle+\langle Y X X Y\rangle) \cos \delta \\
\propto & \left(\Delta A_{\|} \cos ^{2}(\delta)+\Delta A_{\perp}\left(1+\sin ^{2}(\delta)\right)\right) \\
& \pm \cot (\beta)\left(\Delta A_{\|}-\Delta A_{\perp}\right) \cos (\delta)
\end{aligned}
$$

The dependence of the different signals on the angle $\omega$ between the transition dipoles interacting with pump and probe beam is now readily found from well-known expressions for orientational averages: ${ }^{24}$

$$
\begin{aligned}
& A_{\|}+A_{\perp} \propto \frac{2 P_{2}[\cos (\omega)]+10}{45} \\
& A_{\|}-A_{\perp} \propto \frac{2 P_{2}[\cos (\omega)]}{15} .
\end{aligned}
$$

The same proportionality constant applies in both lines. The dependence on pump-probe delay $t$ for molecules that behave like spherical diffusors is readily obtained by multiplying the second order Legendre polynomial $P_{2}$ by $e^{-6 D t}$, where $D$ is the diffusion constant. ${ }^{24}$

Combining Eqs. (A8) and (A14), we arrive at the socalled mystic angle condition $\tan \beta_{M}=1 / 3$, for which $P_{2}$ is eliminated from the signal $\Delta A_{\beta_{M}}$. It thus becomes independent of intramolecular angle and orientational dynamics.

${ }^{1}$ R. Hochstrasser, M. Pereira, P. Share, M. Sarisky, Y. Kim, S. Repinec, R. Sension, J. Thorne, M. Iannone, R. Diller, P. Anfinrud, C. Han, T. Lian, and B. Locke, J. Chem. Sci. 103, 351 (1991).

${ }^{2}$ A. Usman, O. F. Mohammed, E. T. J. Nibbering, J. Dong, K. M. Solntsev, and L. M. Tolbert, J. Am. Chem. Soc. 127, 11214 (2005).

${ }^{3}$ M. Theisen, M. Linke, M. Kerbs, H. Fidder, M. E.-A. Madjet, A. Zacarias, and K. Heyne, J. Chem. Phys. 131, 124511 (2009).

${ }^{4}$ C. V. Shank and E. P. Ippen, Appl. Phys. Lett. 26, 62 (1975).

${ }^{5}$ D. Waldeck, A. J. Cross, D. B. McDonald, and G. R. Fleming, J. Chem. Phys. 74, 3381 (1981).

${ }^{6}$ H. P. Jensen, J. A. Schellman, and T. Troxell, Appl. Spectrosc. 32, 192 (1978).

${ }^{7}$ A. Keston and J. Lospalluto, Fed. Proc. 10, 207 (1951).

${ }^{8}$ D. Che, D. B. Shapiro, R. M. Esquerra, and D. S. Kliger, Chem. Phys. Lett. 224, 145 (1994).

${ }^{9}$ D. S. Alavi, R. S. Hartman, and D. H. Waldeck, J. Chem. Phys. 92, 4055 (1990).

${ }^{10}$ N. F. Scherer, L. D. Ziegler, and G. R. Fleming, J. Chem. Phys. 96, 5544 (1992).

${ }^{11}$ M. Bonmarin and J. Helbing, Chirality 21, E298 (2009).

${ }^{12}$ P. Hamm, R. A. Kaindl, and J. Stenger, Opt. Lett. 25, 1798 (2000).

${ }^{13}$ F. Lumento, V. Zanirato, S. Fusi, E. Busi, L. Latterini, F. Elisei, A. Sinicropi, T. Andruniow, N. Ferre, R. Basosi, and M. Olivucci, Angew. Chem., Int. Ed. 46, 414 (2007).

${ }^{14}$ A. Sinicropi, E. Martin, M. Ryazantsev, J. Helbing, J. Briand, D. Sharma, J. Leonard, S. Haacke, A. Cannizzo, M. Chergui, V. Zanirato, S. Fusi, F. Santoro, R. Basosi, N. Ferre, and M. Olivucci, Proc. Natl. Acad. Sci. U.S.A. 105, 17642 (2008).

${ }^{15}$ See supplementary material at http://dx.doi.org/10.1063/1.3572334 for the description of the synthesis and characterization of the particular NAIP switch used.

${ }^{16}$ J. Briand, O. Bräm, J. Réhault, J. Léonard, A. Cannizzo, M. Chergui, V. Zanirato, M. Olivucci, J. Helbing, and S. Haacke, Phys. Chem. Chem. Phys. 12, 3178 (2009).

${ }^{17}$ M. J. Frisch, G. W. Trucks, H. B. Schlegel et al., GAUSSIAN 03, Gaussian, Inc., Pittsburgh, PA, 2004. 
${ }^{18}$ D. J. Dummer, S. G. Kaplan, L. M. Hanssen, A. S. Pine, and Y. Zong, Appl. Opt. 37, 1194 (1998).

${ }^{19}$ C. Niezborala and F. Hache, J. Opt. Soc. Am. B 23, 2418 (2006).

${ }^{20}$ D. B. Shapiro, R. A. Goldbeck, D. P. Che, R. M. Esquerra, S. J. Paquette, and D. S. Kliger, Biophys. J. 68, 326 (1995).
${ }^{21}$ J. Helbing and M. Bonmarin, J. Chem. Phys. 131, 174507 (2009).

${ }^{22}$ W. Xiong and M. T. Zanni, Opt. Lett. 33, 1371 (2008).

${ }^{23}$ S. S. Andrews, J. Chem. Educ. 81, 877 (2004).

${ }^{24}$ R. M. Hochstrasser, Chem. Phys. 266, 273 (2001). 